\title{
Closing the intention-behaviour gap for sunscreen use and sun protection behaviours
}

Objective: Two studies were conducted with the aim of closing the intentionbehaviour gap for sunscreen use and sun protection behaviours by examining the influence of self-regulatory capacity on the prediction of sunscreen use (Study 1) and self-regulatory capacity and habit on sun protection behaviours (Study 2).

Design: Studies were conducted online. Participants completed questionnaires and cognitive measures at time one and reported behaviour one week later.

Main Outcome Measures: Questionnaires measuring intention and cognitive tasks measuring self-regulatory capacity were administered to 209 university students. One week later, participants reported behaviour. In Study 2, questionnaires measuring intentions and habit and cognitive tasks measuring self-regulatory capacity were administered to 178 university students who reported behaviour one week later.

Results: Intention accounted for $7.1 \%$ of variance in sunscreen use, no measures of self-regulation accounted for variance in behaviour or moderated the intentionbehaviour gap (Study 1). Intention, self-regulatory capacity and habit accounted for $56.1 \%$ of variance in sun protection behaviours (Study 2). Intention, self-regulatory ability and habit predicted behaviour, while habit moderated the intention-behaviour gap.

Conclusion: Interventions aimed at increasing sun protection behaviours should take into account level of intention, self-regulatory capacity and habit. Individuals may benefit from habit formation strategies and self-regulation training.

Keywords: intention-behaviour gap; self-regulation; habit; sun protection behaviours, sunscreen 


\section{Introduction}

Skin cancer is a significant health problem particularly among Caucasian populations. In Australia, skin cancer causes around 1700 deaths each year and costs the health system more to treat than any other form of cancer (Australian Institute of Health and Welfare, 2005). Yet almost all cases are preventable through the performance of appropriate sun protection behaviours (Cancer Council Australia, 2009). Sun protection behaviours include using SPF30+ sunscreen, wearing protective clothing such as a hat, long sleeved shirt and sunglasses, and seeking shade during peak hours of the day (between 10 a.m. and 3 p.m.). Although most individuals are aware of the dangers of sun exposure and how to protect themselves from this danger, many do not sufficiently enact sun protection behaviours (Dobbinson, Jamsen, Francis, Dunlop, \& Wakefield, 2007). Given the personal and public burden of skin cancer, research that aims to explore how individuals translate intention into action for sunscreen use and sun protection behaviours is essential.

\section{Intention - Behaviour Gap}

Theoretical models designed to predict health behaviour, such as the Health Action Process Approach (Schwarzer, 1999) and the Theory of Planned Behaviour (Ajzen, 1991), have repeatedly found that forming an intention to perform a behaviour is beneficial to the actual performance of that behaviour. However, research has indicated that there is an inconsistency between forming a behavioural intention and carrying out the subsequent behaviour (McEachan, Conner, Taylor, \& Lawton, 2011), resulting in a theoretical 'gap' between intention and behaviour (Sniehotta, Scholz, \& Schwarzer, 2005). Consequently, research has attempted to disentangle the relationship between intention and behaviour by testing additional post-intentional variables. For example, both action plans (Sutton, 2008) and implementation intentions (Sheeran \& Orbell, 1999), have been shown to increase the likelihood that intention is translated into action. Similarly, within the literature attempting to 
predict sunscreen use and sun protection behaviours, variables such as time perspective (Schüz \& Eid, 2012), image norms (White et al., 2008) and autonomous motivation (Pavey \& Sparks, 2010) have been shown to influence the relationship between intention and behaviour.

\section{Self-regulatory capacity and health behaviour}

A variable that is inherently linked to the execution of intention is self-regulatory capacity. Self-regulatory capacity refers to the ability to exert control over cognition and emotion in order to organise and direct thinking towards enacting an intended behaviour (Abraham, Sheeran, \& Johnston, 1998). Indeed, specific facets of self-regulatory capacity have been shown to influence the prediction of health behaviours beyond that predicted by intention. For example, Mullan and colleagues have shown that self-regulatory capacity can predict unique variance in a variety of health behaviours including binge drinking (Mullan, Wong, Allom, \& Pack, 2011) and sleep hygiene (Kor \& Mullan, 2011). Importantly, some aspects of self-regulation have been shown to moderate the relationship between intention and behaviour. Allom and Mullan (in press) found that individuals who score lower on an objective measure of impulsivity are more likely to carry out their fruit and vegetable consumption intentions. Similarly, Hall, Fong, Epp and Elias (2008) found that individuals who exhibit superior inhibitory control are more successful in translating intention into action for physical activity and dietary behaviour. Finally, Allan, Johnston, and Campbell (2011) not only found that individual differences in response inhibition predicted snacking behaviour, but that general self-regulatory capacity could account for variance in the size of the intention-behaviour gap for both snacking and fruit and vegetable consumption.

Self-regulatory capacity may be particularly useful for sunscreen use and sun protection behaviours as the primary benefits of these behaviours; avoiding skin cancer (Keeney, McKenna, Fleming, \& McIlfatrick, 2009) and pre-mature aging (Mahler, Kulik, 
Gerrard, \& Gibbons, 2006), are distal. It may be the case that those with a superior selfregulatory capacity are better equipped to keep these future goals in mind while carrying out their intentions in the present. While self-regulatory capacity can be thought of as a general ability to execute intended action, this capacity is made up of several different facets (Suchy, 2009) that may be specifically influential in the execution of sunscreen use and sun protection behaviours. For example, planning ability may be necessary to remember to buy, apply, carry and reapply sunscreen (Craciun, Schüz, Lippke, \& Schwarzer, 2012). Cognitive flexibility, the ability to shift cognitions when the situation changes and adapt behavioural responses accordingly (Spiro, Feltovich, Jacobson, \& Coulson, 1992), may be necessary to ensure than an individual performs sun protection behaviours when unexpectedly required to go into the sun. Finally, low impulsivity would be optimal for focusing on the long term goal of avoidance of skin cancer or pre-mature aging rather than the immediate goal of getting a tan (Murray \& Turner, 2004).

\section{Study 1}

\section{Aims}

The aim of Study 1 is to explore whether self-regulatory capacity predicts the use of sunscreen but also influences the relationship between intention and behaviour. Study 1 is focused only on the prediction of sunscreen use as despite prevention campaigns which advocate the performance of all sun protection behaviours, sunscreen is often used in isolation (Stanton, Janda, Baade, \& Anderson, 2004; Weinstock, Rossi, Redding, Maddock, \& Cottrill, 2000). It was therefore important to address this behaviour separately, while the cluster of sun protection behaviours will be addressed in Study 2. Finally, the study will use cognitive tasks to objectively measure the three facets of self-regulation that are hypothesised to be associated with sun protection behaviours: planning ability, cognitive flexibility and impulsivity. 
It was firstly hypothesised that intention to use sunscreen will predict behaviour. It was also expected that self-regulatory capacity will account for additional variance in sunscreen use such that individuals with higher planning ability, higher cognitive flexibility, and lower impulsivity will be more likely to use sunscreen. Finally, self-regulatory capacity was expected to moderate the intention-behaviour relationship such that those with higher planning ability, higher cognitive flexibility and lower impulsivity, will be more likely to translate intention into action.

\section{Method}

\section{Participants}

The sample consisted of 218 students enrolled in an undergraduate psychology course at an Australian University. After two measurement points of data collection the attrition rate was 4.1\% leaving a total of 209. The mean age was 20.06 years $(S D=4.39)$, ranging from 16 to 45 years and the majority of the sample were female $(75 \% ; n=157)$. The participants were recruited using an online registration system and received course credit for their participation. The University Human Research Ethics Committee approved the study.

\section{Procedure}

All parts of the study were completed online in Sydney, during April of 2010. Rainfall during this month was lower than average, with only 10 out of 30 days experiencing rainfall. Further, the temperature was on average 20.2 degrees Celsius, making the likelihood of outdoor activity high. Questionnaires were completed through LimeSurvey software and selfregulatory tasks were administered using Inquisit 3.0 by Millisecond Software. At baseline, participants completed measures of intention and three cognitive tasks which measured different facets of self-regulatory capacity: planning ability, cognitive flexibility and impulsivity. After one week participants completed a questionnaire that measured sunscreen use. 


\section{Measures}

Intention to use sunscreen was calculated as the mean of three items on a 7-point Likert scale ( 1 = strongly disagree, 7 = strongly agree), e.g. "I intend to use sunscreen when I go in the sun.” The three items had a Cronbach's alpha of .93.

Behaviour was measured one week later by two items: "Did you use sunscreen over the past week when you went in the sun?” (yes/no) and “On how many days did you apply sunscreen over the past week when you went in the sun?” The two items had a Spearman's rho of .94, $p<.01$.

Planning ability was measured using a computerised Tower of London (TOL) task, which is based on the original task developed by Shallice (1982) and has been validated for a normal population by Kafer and Hunter (1997). Participants were presented with a starting configuration of three disks on three pegs and were required to move the disks in as few moves as possible to match an end configuration. The task consisted of 12 trials which increased in difficulty. Planning ability was measured as the mean time spent preplanning for each problem (Berg \& Byrd, 2002), where a higher score indicated greater planning ability.

Cognitive flexibility was measured by a computerised version of the Wisconsin Card Sorting Task (WCST), which was based on the original task described by Grant and Berg (1948). Imaging studies of nonclinical populations show that this task can indicate individual differences in cognitive function (Wang, Kakigi, \& Hoshiyama, 2001). Participants were required to match a stimulus card to one of four options based on one of three properties: symbol on card, colour of symbol, or number of symbols. Once the rule had been solved correctly four times in a row, the property by which the cards were to be sorted changed. The task consisted of nine blocks: a block per property, repeated three times. The minimum number of trials was 36 given that a participant had to respond correctly four times in a row to progress to the next block. The variable of interest was the number of 'perseverance' 
errors. Such errors occur when the rule has changed yet participants continue to respond in a way that is appropriate to the previous rule. Less error indicated superior cognitive flexibility. Impulsivity was measured by a computerised version of the Iowa Gambling Task (IGT) based on that described by Bechara, Damasio, Damasio and Anderson (1994). This task has been previously used to measure individual differences in decision making in normal populations (Suhr \& Hammers, 2010). Participants were required to place bets on one of four decks of cards which each resulted in different degrees of loss or gain. Two decks were advantageous in that participants received a larger net gain even though initially winnings seemed conservative. The task consisted of 50 trials. A greater amount of money remaining at the end of the task indicated less impulsivity in decision making (Brand, Recknor, Grabenhorst, \& Bechara, 2007).

\section{Analysis}

Data was analysed using IBM SSPS Statistics 19. Hierarchical regression analyses were performed to determine whether intention and facets of self-regulatory capacity could predict sunscreen use and whether the interactions between intention and facets of self-regulatory capacity accounted for additional variance in sunscreen use. Cross-products between mean centred self-regulatory variables and mean centred intention were calculated and entered into the regression to test for moderation (Keith, 2006).

\section{Results}

Of the 209 participants who completed the study, 34.5\% $(n=72)$ used sunscreen over the week the study was carried out. Means, standard deviations and correlations of the variables are reported in Table 1. Intention was positively correlated with behaviour. However, none of the self-regulatory capacity variables correlated significantly with behaviour or intention.

INSERT TABLE 1 HERE 
Regression analyses were conducted in order to test whether intention and the additional self-regulatory capacity variables predicted sunscreen use. Intention was entered first and was found to significantly account for $6.6 \%$ of the variance in sunscreen use $\left(F_{1,207}\right.$ $=14.60, p<.01)$. All self-regulatory measures were simultaneously entered in the next step, accounting for an additional .3\% of the variance in sunscreen use, which was not significant $\left(F_{3,204}=.23, p=.88\right)$. The products of centred intention and centred self-regulatory measures were then entered in three consecutive steps to test for moderation effects. Neither planning ability $\left(F_{1,203}=.30, p=.58\right)$, cognitive flexibility $\left(F_{1,202}=.08, p=.78\right)$, nor impulsivity $\left(F_{1}\right.$, $201=.14, p=.71$ ), were found to significantly moderate the relationship between intention and behaviour. The overall model accounted for $7.1 \%$ of the variance in sunscreen use (see Table 2).

\section{INSERT TABLE 2 HERE}

\section{Discussion}

The aim of this study was explore whether self-regulatory capacity predicted sunscreen use and influenced the relationship between intention and behaviour. The hypothesis that intention would predict future sunscreen use was supported; intention accounted for $6.6 \%$ of the variance in sunscreen use, indicating that if intention to use sunscreen is high, the performance of this behaviour is more likely. Previous research examining whether intention can predict sunscreen use is varied. For example, Jackson and Aiken (2006) did not find that intention to use sunscreen significantly predicts behaviour, while Jones, Abraham, Harris, Schulz, and Chrispin (2001) found intention accounted for 30\% of the variance in sunscreen use. The discrepancy in findings may be due to inconsistent measurement of intention and behaviour. In the current study, the items measuring these variables did not explicitly follow the 'TACT' principles (Ajzen, 2002), whereby only the target and action associated with the behaviour were specified and context and time was not included. This was done to simplify 
the items in order to avoid confusion. Also, it was initially thought that because the need to use sunscreen is variable, it would be difficult to specify a time and context. However, future research should attempt to satisfy all TACT principles in order to accurately measure intention and behaviour.

Similar to previous findings (Myers \& Horswill, 2006), a large amount of variance in sunscreen use remains unaccounted for. While it was expected that post-intentional variables would increase the accounted for variance in behaviour, no measures of self-regulatory capacity explained additional variance in sunscreen use or moderated the relationship between intention and behaviour. This was contrary to previous studies which found that individual differences in self-regulatory capacity can predict health behaviours and moderate the relationship between intention and behaviour (Allom \& Mullan, in press).

To test if there was enough power to detect the effect of self-regulatory capacity on behaviour, a sensitivity analysis was conducted using G-Power software (Faul, Erdfelder, Buchner, \& Lang, 2009). The analysis revealed that with a sample size of 209, and even with seven total predictors, the study was sufficiently powered to detect R-square changes with an effect size of $\mathrm{f}^{2}>0.11$. This is classified as a 'small' to 'medium' effect (Cohen, 2003). Therefore, the null results are unlikely to be due to lack of power. It is possible that the tasks used to measure self-regulatory capacity were too general, and instead should have been tailored to reflect the behaviour of interest. For example, rather than measuring general planning ability, it may have been more useful to measure planning ability for sunscreen use. Future research may benefit from the development of such behaviour-specific tasks.

Additionally, sunscreen use may be a relatively simple behaviour, the execution of which may not require a superior self-regulatory capacity. It is possible that for the more complex array of behaviours involved in sun protection behaviour, having a greater selfregulatory capacity will be beneficial. Alternatively, given that there is an appearance-based 
motivation to use sunscreen, it may be the case that individual differences in self-regulation are not as useful for the prediction of sunscreen use as other variables such as image norms (Mahler, et al., 2006; White, et al., 2008). Further, it may be the case that automatic processes such as habit influence sunscreen use. As sunscreen application is a repetitive behaviour, the performance of this behaviour may have become routine for some individuals, which may partially explain why intention does not account for a great deal of variance in this behaviour. Individuals who have formed a habit may no longer need to set explicit goals in order to carry out a behaviour (Ouellette \& Wood, 1998), and if goal setting is not necessary, selfregulation, which assists in the execution of goals, may not be required (Orbell \& Verplanken, 2010).

\section{Study 2}

As described previously, although sunscreen use is the most common form of sun protection and often used in isolation from the remaining behaviours, adequate sun protection involves performing the full range of sun protection behaviours. As such, the aim of Study 2 was to predict this cluster of behaviours and explore the relationship between intention and behaviour for sun protection behaviours. Although self-regulatory capacity did not predict behaviour in Study 1, it is expected that this variable may play a role in the prediction of a more complex set of behaviours.

\section{Habit strength}

Additionally, it is suggested that habit strength may be a useful determinant of behaviour. Considering the finding that conscious processes such as intention did not account for a large amount of variance in sunscreen use, automatic processes such as habit may play a role in sun protection behaviours. Research has shown that accounting for the role of habit in addition to intention has led to better prediction of behaviour (Verplanken, Aarts, Van Knippenberg, \& Moonen, 1998). Allom and Mullan (in press) found an interaction between habit and 
intention such that intentions were only significantly related to fruit and vegetable consumption when habit strength was weak. In terms of predicting sun protection behaviours, the addition of habit may be useful as the performance of these behaviours is required daily and may have become part of an individual's routine.

\section{Aims}

The aim of Study 2 was to disentangle the intention-behaviour gap for the cluster of behaviours referred to as sun protection behaviours, by measuring self-regulatory capacity and habit strength. It is hypothesised that intention, self-regulatory capacity and habit will predict sun protection behaviours such that higher levels of these variables will lead to greater performance of behaviour. Further, self-regulatory capacity and habit are both expected to moderate the relationship between intention and behaviour such that those with higher levels of planning ability, higher cognitive flexibility and lower impulsivity will be more likely to translate intention into action, whereas for those with a greater habit strength, behaviour will not be a function of intention. As habit encompasses more than the frequency of past behaviour (Verplanken \& Orbell, 2003), past behaviour will be controlled for in order to distinguish the unique role of habit. It is hypothesised that the effect of habit will be present over and above the effect of past behaviour. Further, to address one of the limitations of Study 1 , measures of intention and behaviour were constructed with the two additional TACT principles of context and time (Ajzen, 2002).

\section{Method}

\section{Participants}

The sample consisted of 227 students enrolled in a first year psychology course. After two measurement points the attrition rate was $21.6 \%$ leaving a total of 178 participants. The high attrition rate was due to technical issues experienced when completing the computer tasks. The mean age was 19.41 years $(S D=4.00$ years), ranging from 17 to 44 years and the 
majority of participants were female $(74 \% ; n=132)$. The participants were recruited using an online registration system and received course credit for their participation. The study was approved by the University’s Human Research Ethics Committee.

\section{Procedure}

As with Study 1, all parts of Study 2 took place online, in Sydney, during May of 2011.

Rainfall during this month was lower than average, with only 11 out of 31 days experiencing rainfall. Further, the temperature was on average 19.5 degrees Celsius, making the likelihood of outdoor activity high. Questionnaires were administered through LimeSurvey software and self-regulatory tasks were conducted using Inquisit 3.0 by Millisecond Software. Participants were informed that sun protection behaviours involved '...using SPF30+ sunscreen, wearing protective clothing such as a hat, long sleeved shirt and sunglasses, or seeking shade during peak hours of the day (between 10 a.m. and 3 p.m.)'. After one week, participants completed a follow-up questionnaire that measured sun protection behaviours.

\section{Measures}

Intention was measured by the mean of three items on a 7-point Likert scale $(1=$ strongly disagree, 7 = strongly agree), e.g. "I intend to perform sun protection behaviours every time I go in the sun for more than 10 minutes over the next week.” The three items had a Cronbach's alpha of .91.

Past behaviour was measured with a 7-point item (1 = never, 7 = always), “Think about the past week, how often did you perform sun protection behaviours every time you went in the sun for more than 10 minutes”.

Behaviour was measured by firstly asking participants to indicate whether they performed sun protection behaviours when in the sun for more than 10 minutes over the past week $(0=$ no, 1 = yes $)$. Secondly, participants were asked to report their behaviour by indicating how often they were in the sun ("How often did you go in the sun for more than 10 
minutes over the past week?”) and of these times how often did they use sun protection behaviours ("How often did you use sun protection behaviours when you went in the sun for more than 10 minutes over the past week?”), for each day of the week. From this a percentage was calculated indicating the proportion of times participants used sun protection when in the sun (Hillhouse, Adler, Drinnon, \& Turrisi, 1997). The two items had a Spearman’s rho of .83 .

Self-regulatory capacity: Planning ability (TOL), cognitive flexibility (WCST) and impulsivity (IGT) were measured using the same method as in Study 1.

Habit was measured by the Self Report Habit Index (Verplanken \& Orbell, 2003). Participants responded to the stem "'Performing sun protection behaviours every time I go in the sun for more than 10 minutes is something...’ which was followed by the 12 items of the scale designed to measure intentionality, controllability, awareness, and mental efficiency, e.g. “"...that belongs to my daily routine,” and “...that would require effort not to do it." Responses were given on 7-point Likert Scales ( 1 = strongly disagree, 7 = strongly agree). Higher scores indicated that sun protection behaviours were more likely to be influenced by habit. The 12 items had a Cronbach’s alpha of .97.

\section{Analysis}

Data was analysed using IBM SPSS Statistics 19. Hierarchical regression analyses were performed to determine whether intention, habit and self-regulatory capacity predict sun protection behaviours over and above past behaviour. Variables were mean-centred and cross-products between variables and mean centred intention were calculated and entered into the regression to test for moderation effects (Keith, 2006).

\section{Results}

The 178 participants in this sample used some form of sun protection on average $47.57 \%$ of the times they were in the sun for longer than 10 minutes. Means, standard deviations and 
correlations of variables are reported in Table 3. Intention, past behaviour and habit correlated positively with actual behaviour, indicating that the more one intends to, or has carried out the behaviour in the past, the more likely they are to perform the behaviour in the future. Scores on the WCST, which measured cognitive flexibility, correlated negatively with intention, past behaviour, habit, and actual behaviour. As lower scores indicated greater cognitive flexibility, the negative correlation revealed that greater cognitive flexibility was associated with an increased likelihood of intending to perform sun protection behaviours, of having performed these behaviours in the past, and of performing them in the future.

\section{INSERT TABLE 3 HERE}

\section{Predicting Behaviour}

As cognitive flexibility was the only variable significantly correlated with intention and behaviour, only this facet of self-regulatory capacity was used in the regression analysis to predict behaviour. This was done to preserve statistical power given that a greater number of predictors in a regression model decreases power to detect effects (Keith, 2006).

Intention was entered first and accounted for $23.6 \%$ of the variance in sun protection behaviour $\left(F_{1,176}=54.46, p<.01\right)$. Past behaviour was entered next to control for the effect of this variable on subsequent variables and was found to add $10.7 \%$ to the prediction of sun protection behaviour $\left(F_{1,175}=28.58, p<.01\right)$. Cognitive flexibility was found to account for $10.4 \%$ of additional variance in behaviour such that those with fewer errors on the WCST (or higher cognitive flexibility) were more likely to perform sun protection behaviours $\left(F_{1,174}=\right.$ 32.85, $p<.01$ ). Habit added a further $8.5 \%$ over and above the effect of past behaviour such that those with stronger habits were more likely to perform the behaviour $\left(F_{1,173}=31.30, p\right.$ $<$.01). Cognitive flexibility was not found to moderate the relationship between intention and behaviour $\left(F_{1,172}=2.94, p=.09\right)$. Finally, habit moderated the intention-behaviour gap such that individuals with higher levels of habit were likely to carry out the behaviour despite their 
intentions $\left(t_{172}=0.03, p=0.77\right)$, whilst those with lower habit strength were more likely to perform the behaviour if they intended to $\left(t_{172}=2.06, p=0.04\right)$; see Figure 1 . The interaction between habit and intention contributed a further $2.1 \%$ to the explained variance in behaviour $\left(F_{1,171}=8.08, p<.01\right)$. The overall model accounted for $56.1 \%$ of the variance in sun protection behaviour (see Table 4).

\section{INSERT FIGURE 1 HERE}

INSERT TABLE 4 HERE

\section{Discussion}

The aim of this study was explore the relationship between intention and behaviour for sun protection behaviours through the inclusion of self-regulatory capacity and habit. It was hypothesised that intention would predict behaviour and that self-regulatory capacity and habit would have direct and indirect effects on behaviour. Intention, habit and one facet of self-regulatory capacity predicted behaviour and habit moderated the intention behaviour gap. However, self-regulatory capacity did not influence the relationship between intention and behaviour.

The present research found that cognitive flexibility predicts the performance of sun protection behaviours. The ability to shift cognition and consequent behaviour to suit the environment, for example when the sun reappears on a rainy day, is particularly important to sun protection behaviours as the need to carry out these behaviours varies depending on the weather. In comparison, impulsivity; measured by the IGT, was not found to be predictive of sun protection behaviours. Choosing to not use sun protection may not constitute an impulsive behaviour as the outcome of this choice is distal. Further, planning ability; measured by the TOL, was not found to be predictive of sun protection behaviours. This finding was unexpected particularly given research that has shown interventions aimed to increase planning, such as the development of implementation intentions, can improve the 
performance of sun protection behaviours (Craciun, et al., 2012). However, the present study conceptualised planning as an ability, rather than an intervention strategy, which could explain the inconsistency in these results.

Habit was found to have a robust effect on future behaviour even when past behaviour was accounted for. This is consistent with previous research that found habit was a strong predictor of future behaviour (Ouellette \& Wood, 1998) and implies that sun protection behaviours may be under habitual control. It is believed that when a particular behaviour is repeated frequently and consistently in a similar situation, it eventually becomes automatic or habitual (Brickell, Chatzisarantis, \& Pretty, 2006). The behaviour then requires less conscious attention and effort to be executed. For many individuals sun protection behaviours, such as sunscreen use, become part of a daily routine. These behaviours are likely to be cued by recurring features of the environment or stable contexts (Ouellette \& Wood, 1998). For sun protection behaviours, there may be a variety of internal and external cues that prompt people to perform these behaviours. For example, walking to work or having a lunch break in the sun may have been associated with sunscreen use in the past and now automatically triggers sunscreen use.

As well as investigating the predictive effects of habit, the present study also found that habit moderated the association between intention and behaviour. Intention to perform sun protection behaviours appeared to have little impact on the behaviour of individuals with high habit strength, compared to those individuals’ with low habit strength for whom intention was predictive of behaviour. This finding is similar to Verplanken, et al. (1998) who found that intention interacted with habit to predict behaviour. This relationship suggests two avenues that interventions may take to improve health behaviour: increase habit strength or enhance intention. For example, for someone who has not performed the behaviour in the past, it may be beneficial to increase one's intentions. While someone who has experience 
with the behaviour may benefit from interventions that increase automaticity. Finally, these strategies may be used in conjunction whereby once an individual has a strong intention to perform the behaviour, habit strength can be bolstered.

\section{General Discussion}

Two studies were carried out with the aim of improving the prediction of sunscreen use and sun protection behaviours using the post intentional variables of self-regulatory capacity and habit strength. For sunscreen use, intention did not account for a great deal of the variance in behaviour, most likely due to the inconsistency in measures of intention and behaviour. However, for sun protection behaviours, intention accounted for a sizable amount of variance in behaviour. The results from Study 1 do not support the addition of self-regulatory capacity as a direct predictor of behaviour or as a moderator of the relationship between intention and behaviour as no self-regulatory measures added to the explained variance in sunscreen use. However, the findings from Study 2 support the inclusion of self-regulatory capacity as an addition to intention as cognitive flexibility predicted sun protection behaviours. The discrepancy between self-regulatory capacity as a predictor of behaviour in Study 1 and Study 2 is firstly likely due to the different outcome variables that were measured; sunscreen use versus sun protection behaviours. However, it may also be the case that self-regulation is needed for the full range of sun protection behaviours as several behaviours are more difficult to carry out than one.

Further, habit strength appears to play a role in performing sun protection behaviours as habit directly added to the accounted for variance in this behaviour and moderated the relationship between intention and behaviour. These results are similar to that predicted by a relatively new model of health behaviour, referred to as Temporal self-regulation theory (Hall \& Fong, 2007). It is suggested that for behaviours where the benefits do not occur immediately, post-intentional variables influence the prediction of these behaviours. 
Specifically, self-regulation and constructs similar to habit strength are required to translate intention into action. This is similar to previous research that suggests that intention and past behaviour may be differentially influential in the prediction of behaviour depending upon the favourableness of behavioural circumstances (Ouellette \& Wood, 1998). Future research should take into account whether or not one finds the circumstances surrounding a particular behaviour to be unfavourable or favourable (immediate versus distal benefits), as an important influence on the performance of behaviour.

\section{Implications}

These results have implications for designing interventions for behaviour change. The current research has identified factors on which intervention efforts should focus to increase the frequency of sun protection behaviours. The role of habit in sun protection behaviours implies that interventions need to target development of performing these behaviours as a habit. Habits can be formed by linking already automatic behaviours with a target behaviour (Lally \& Gardner, in press), for example: pairing locking the front door with putting on a hat may lead to automatic performance of sun protection behaviours.

Understanding self-regulatory capacity gives an alternative strategy for individuallevel intervention and health promotion (Abraham, et al., 1998). Self-regulatory capacity is said to be like a muscle such that it can become exhausted but also strengthened over time with repeated exercises of self-control (Muraven, Baumeister, \& Tice, 1999). Recently, studies aiming to train self-regulatory capacity have shown that some self-regulatory abilities, such as working memory, can be improved by repeated performance on cognitive tasks similar to those used in the present research (see Klingberg, 2010 for a review). Houben, Wiers and Jansen (2011) found that this improvement in self-regulatory capacity was not confined to the task on which participants were trained, but rather, transferred to another task measuring working memory and even led to improvements in health behaviour. Future 
research could focus on training participants on a cognitive flexibility task in order to determine whether this training transfers to improvements in sun protection behaviours.

\section{Limitations}

There are limitations to the present studies that must be acknowledged. As previously described, Study 1 would have benefited from the use of items that were developed with the TACT principles in mind. Additionally, measurement of habit and past behaviour in this study would have indicated the role of these variables for the specific behaviour of sunscreen use. Secondly, due to the measurement of a cluster of behaviours in Study 2, it unclear whether self-regulation and habit predict all behaviours in the cluster or only certain behaviours. Future studies aiming to explain the intention-behaviour gap for sun protection behaviours should have multiple outcome variables targeting each sun protection behaviour as well as the behavioural cluster. Additionally, the studies were carried out online, which may have reduced the reliability of responses on self-regulatory tasks. Future studies could conduct the study in the lab to ensure tasks are completed in a controlled environment.

Additionally, an interval of one week for baseline and follow up measures was used for both studies. It is possible that one week did not allow enough time to confirm the long term efficacy of the TPB or to explore the role of self-regulation as a predictor of behaviour over a longer time period. It may be the case that, over an extended time frame, the effects of self-regulation are negated by the effects of intention. Alternatively, individual differences in self-regulation may explain more variance in behaviour over a longer period of time. These issues may be resolved in future studies that use a longitudinal approach.

The predominantly female samples that were used for both studies may have influenced the results and the conclusions that can be drawn. Specifically, findings have suggested that women are more knowledgeable about skin cancer and also perceive a higher susceptibility to skin cancer than men, thus women may be more inclined to engage in sun 
protection behaviours (Arthey \& Clarke, 1995). Additionally, some women who wear makeup may not be aware that certain products include sun protection. As a result, some women may not intend to wear sun protection, but do so anyway, or may not be aware that they are wearing sun protection and do not accurately report this behaviour. Future studies may benefit from the inclusion of information regarding sun protection in make-up as well as items to measure the intention to use such products as sun protection and actual use.

Related to this, an interesting avenue for future research would be to classify participants into the four categories of intention (inclined/disinclined) and action (acted/did not act) as described by Sheeran (2002) in order to explore which group is more likely to be performing behaviour as a function of self-regulatory capacity and/or habit strength. For example, self-regulatory capacity may be more influential for inclined abstainers who intend to perform a behaviour but do not, while habit may be predictive of disinclined actors, who do not intend to, yet perform the behaviour anyway. Finally, perhaps a more careful examination of why participants intend to use sun protection behaviours (avoidance of skin cancer or appearance based) may influence whether or not intention is translated into action. Future research could explore the effect of motivation on the intention-behaviour gap for sun protection behaviours.

\section{Conclusions}

While self-regulatory capacity did not increase the prediction of sunscreen use or aid in disentangling the intention-behaviour gap for this behaviour, Study 2 revealed that selfregulatory capacity was related to the cluster of behaviours known as sun protection behaviours. Additionally, habit was found to directly predict sun protection behaviour as well as moderate the intention behaviour gap. These results add to the growing literature on the intention behaviour gap and suggest that self-regulatory capacity and habit strength are important variables for the prediction of some health behaviours and indicate possible ways 
by which health behaviours can be improved. This is particularly relevant given the burden of skin cancer and the lack of adherence to sun protection behaviours. 


\section{Acknowledgements}

This research was funded by WorkCover Authority of New South Wales under an Australian Research Council grant. The authors would like to thank the reviewers for their time and helpful critique of the original manuscript. Finally, thank you to Tyler Clark for assisting with data collection and the University of Sydney Health Psychology Lab Group for their advice on this paper. 


\section{References}

Abraham, C., Sheeran, P., \& Johnston, M. (1998). From health beliefs to self-regulation: Theoretical advances in the psychology of action control. Psychology \& Health, 13, 569-591.

Ajzen, I. (1991). The theory of planned behavior. Organizational Behavior and Human Decision Processes, 50, 179-211.

Ajzen, I. (2002). Constructing a TPB questionnaire: Conceptual and methodological considerations. http://people.umass.edu.ajzen/pdf/tpb.measurement.pdf Retrieved 23rd March 2010

Allan, J., Johnston, M., \& Campbell, N. (2011). Missed by an inch or a mile? Predicting size of intention-behaviour gap from measures of executive control. Psychology \& Health, 26, 635-650.

Allom, V., \& Mullan, B. (in press). Self-regulation versus habit: The influence of self-schema on fruit and vegetable consumption. Psychology \& Health.

Arthey, S., \& Clarke, V. A. (1995). Suntanning and sun protection: a review of the psychological literature. Social science \& medicine, 40, 265-274.

Australian Institute of Health and Welfare. (2005). Health system expenditures on cancer and other neoplasms in Australia, 2000-01. Canberra: AIHW (Health and Welfare Expenditure Series no. 22).

Bechara, A., Damasio, A., Damasio, H., \& Anderson, S. (1994). Insensitivity to future consequences following damage to human prefrontal cortex. Cognition, 50, 7-15.

Berg, W. K., \& Byrd, D. L. (2002). The Tower of London spatial problem-solving task: Enhancing clinical and research implementation. Journal of Clinical and Experimental Neuropsychology, 24, 586-604. 
Brand, M., Recknor, E. C., Grabenhorst, F., \& Bechara, A. (2007). Decisions under ambiguity and decisions under risk: Correlations with executive functions and comparisons of two different gambling tasks with implicit and explicit rules. Journal of Clinical and Experimental Neuropsychology, 29, 86-99.

Brickell, T. A., Chatzisarantis, N. L. D., \& Pretty, G. M. (2006). Using past behaviour and spontaneous implementation intentions to enhance the utility of the theory of planned behaviour in predicting exercise. British Journal of Health Psychology, 11, 249-262.

Cancer Council Australia. (2009). Skin cancer prevention: A blue chip investment in health. NSW: The Australasian College of Dermatologists.

Cohen, J. (2003). Applied multiple regression/correlation analysis for the behavioral sciences (Vol. 1): Lawrence Erlbaum.

Craciun, C., Schüz, N., Lippke, S., \& Schwarzer, R. (2012). A Mediator Model of Sunscreen Use: A Longitudinal Analysis of Social-Cognitive Predictors and Mediators. International Journal of Behavioral Medicine, 19, 65-72.

Dobbinson, S., Jamsen, K. M., Francis, K., Dunlop, S., \& Wakefield, M. A. (2007). Skin cancer prevention knowledge, attitudes and beliefs among Australians in summer 2006-07 and comparison with 2003-04 in the context of the first national mass media campaign. Prepared for the Australian Government Department of Health and Ageing, and The Cancer Council Australia, Melbourne: Unpublished Manuscript.

Faul, F., Erdfelder, E., Buchner, A., \& Lang, A. G. (2009). Statistical power analyses using G* Power 3.1: Tests for correlation and regression analyses. Behavior Research Methods, 41, 1149-1160.

Grant, D., \& Berg, E. (1948). A behavioral analysis of degree of reinforcement and ease of shifting to new responses in a Weigl-type card-sorting problem. Journal of Experimental Psychology, 38, 404-411. 
Hall, P., \& Fong, G. (2007). Temporal self-regulation theory: A model for individual health behavior. Health Psychology Review, 1, 6-52.

Hall, P., Fong, G., Epp, L., \& Elias, L. (2008). Executive function moderates the intentionbehavior link for physical activity and dietary behavior. Psychology \& Health, 23, 309-326.

Hillhouse, J. J., Adler, C. M., Drinnon, J., \& Turrisi, R. (1997). Application of Azjen's theory of planned behavior to predict sunbathing, tanning salon use, and sunscreen use intentions and behaviors. Journal of Behavioral Medicine, 20, 365-378.

Houben, K., Wiers, R. W., \& Jansen, A. (2011). Getting a Grip on Drinking Behavior. Psychological Science, 22, 968-975.

Jackson, K. M., \& Aiken, L. S. (2006). Evaluation of a multicomponent appearance-based sun-protective intervention for young women: uncovering the mechanisms of program efficacy. Health Psychology, 25, 34.

Jones, F., Abraham, C., Harris, P., Schulz, J., \& Chrispin, C. (2001). From knowledge to action regulation: Modeling the cognitive prerequisites of sun screen use in Australian and UK samples. Psychology and Health, 16, 191-206.

Kafer, K., \& Hunter, M. (1997). On testing the face validity of planning/problem-solving tasks in a normal population. Journal of the International Neuropsychological Society, 3, 108-119.

Keeney, S., McKenna, H., Fleming, P., \& McIlfatrick, S. (2009). Attitudes, knowledge and behaviours with regard to skin cancer: a literature review. European journal of oncology nursing: the official journal of European Oncology Nursing Society, 13, 29.

Keith, T. (2006). Multiple regression and beyond: Boston: Allyn \& Bacon.

Klingberg, T. (2010). Training and plasticity of working memory. Trends in Cognitive Sciences, 14, 317-324. 
Kor, K., \& Mullan, B. A. (2011). Sleep hygiene behaviours: An application of the theory of planned behaviour and the investigation of perceived autonomy support, past behaviour and response inhibition. Psychology \& Health, 26, 1208-1224.

Lally, P., \& Gardner, B. (in press). Promoting habit formation. Health psychology Review. Mahler, H. I. M., Kulik, J. A., Gerrard, M., \& Gibbons, F. X. (2006). Effects of two appearance-based interventions on the sun protection behaviors of southern California beach patrons. Basic and Applied Social Psychology, 28, 263-272.

McEachan, R. R. C., Conner, M., Taylor, N. J., \& Lawton, R. J. (2011). Prospective prediction of health-related behaviours with the theory of planned behaviour: a metaanalysis. Health Psychology Review, 5, 97-144.

Mullan, B., Wong, C., Allom, V., \& Pack, S. L. (2011). The role of executive function in bridging the intention-behaviour gap for binge-drinking in university students. Addictive Behaviors, 36, 1023-1026.

Muraven, M., Baumeister, R. F., \& Tice, D. M. (1999). Longitudinal improvement of selfregulation through practice: Building self-control strength through repeated exercise. Journal of Social Psychology, 139, 446-457.

Murray, C. D., \& Turner, E. (2004). Health, risk and sunbed use: a qualitative study. Health, Risk \& Society, 6, 67-80.

Myers, L. B., \& Horswill, M. S. (2006). Social cognitive predictors of sun protection intention and behavior. Behavioral Medicine, 32, 57-63.

Orbell, S., \& Verplanken, B. (2010). The automatic component of habit in health behavior: Habit as cue-contingent automaticity. Health Psychology, 29, 374.

Ouellette, J., \& Wood, W. (1998). Habit and intention in everyday life: The multiple processes by which past behavior predicts future behavior. Psychological Bulletin, 124, 54-74. 
Pavey, L. J., \& Sparks, P. (2010). Autonomy and reactions to health-risk information. Psychology and Health, 25, 855-872.

Schüz, N., \& Eid, M. (2012). Beyond the usual suspects: target group-and behavior-specific factors add to a theory-based sun protection intervention for teenagers. Journal of Behavioral Medicine, 1-12.

Schwarzer, R. (1999). Self-regulatory processes in the adoption and maintenance of health behaviors. Journal of health psychology, 4, 115-127.

Shallice, T. (1982). Specific impairments of planning. Philosophical Transactions of the Royal Society of London. Series B, Biological Sciences, 298, 199-209.

Sheeran, P. (2002). Intention-behavior relations: A conceptual and empirical review. European review of social psychology, 12, 1-36.

Sheeran, P., \& Orbell, S. (1999). Implementation intentions and repeated behaviour: Augmenting the predictive validity of the theory of planned behaviour. European Journal of Social Psychology, 29, 349-369.

Sniehotta, F., Scholz, U., \& Schwarzer, R. (2005). Bridging the intention-behaviour gap: Planning, self-efficacy, and action control in the adoption and maintenance of physical exercise. Psychology \& Health, 20, 143-160.

Spiro, R. J., Feltovich, P. J., Jacobson, M. J., \& Coulson, R. L. (1992). Cognitive flexibility, constructivism, and hypertext: Random access instruction for advanced knowledge acquisition in ill-structured domains. In T. M. Duffy (Ed.), Constructivism and the technology of instruction: A conversation (pp. 57-75). New Jersey: Lawrence Erlbaum Associates.

Stanton, W. R., Janda, M., Baade, P. D., \& Anderson, P. (2004). Primary prevention of skin cancer: a review of sun protection in Australia and internationally. Health Promotion International, 19, 369-378. 
Suchy, Y. (2009). Executive functioning: Overview, assessment, and research issues for nonneuropsychologists. Annals of Behavioral Medicine, 37, 106-116.

Suhr, J., \& Hammers, D. (2010). Who fails the Iowa Gambling Test (IGT)? Personality, neuropsychological, and near-infrared spectroscopy findings in healthy young controls. Archives of Clinical Neuropsychology, 25, 293-302.

Sutton, S. (2008). How does the health action process approach (HAPA) bridge the intentionbehavior gap? An examination of the model's causal structure. Applied Psychology, 57, 66-74.

Verplanken, B., Aarts, H., Van Knippenberg, A., \& Moonen, A. (1998). Habit versus planned behaviour: a field experiment. British Journal of Social Psychology, 37, 111-128.

Verplanken, B., \& Orbell, S. (2003). Reflections on past behavior: A self-report index of habit strength. Journal of Applied Social Psychology, 33, 1313-1330.

Wang, L., Kakigi, R., \& Hoshiyama, M. (2001). Neural activities during Wisconsin Card Sorting Test--MEG observation. Cognitive Brain Research, 12, 19-31.

Weinstock, M. A., Rossi, J. S., Redding, J. A., Maddock, J. E., \& Cottrill, S. D. (2000). Sun protection behaviors and stages of change for the primary prevention of skin cancers among beachgoers in southeastern New England. Annals of Behavioral Medicine, 22, 286-293.

White, K. M., Robinson, N. G., Young, R. M. D., Anderson, P. J., Hyde, M. K., Greenbank, S., et al. (2008). Testing an extended theory of planned behaviour to predict young people's sun safety in a high risk area. British Journal of Health Psychology, 13, 435448. 
Table 1. Descriptive analysis of sunscreen use for Study 1: means, standard deviations and bivariate correlations, $\mathrm{N}=209$.

\begin{tabular}{llllllll}
\hline & $M$ & SD & INT & TOL & WCST & IGT & SUN \\
\hline INT & 5.84 & 1.38 & - & -.06 & -.07 & -.02 & $.24^{* *}$ \\
TOL & 1.27 & .08 & - & - & -.01 & -.05 & -.13 \\
WCST & 43.88 & 26.82 & - & - & - & .04 & -.01 \\
IGT & 1665.90 & 631.76 & - & - & - & - & .07 \\
SUN & 1.52 & 2.36 & - & - & - & - & - \\
\hline
\end{tabular}

Note: INT = intention, TOL = planning ability measured by average time spent preplanning (seconds) on Tower of London Task, WCST = cognitive flexibility measured by number of perseverance errors on the Wisconsin Card Sort Task, IGT = impulsivity measured by remaining play money (\$) on Iowa Gambling Task, SUN = sunscreen use (number of days over study week), ${ }^{* *} p<.01,{ }^{*} p<.05$. 
Table 2. Final step of hierarchical regression analysis for Study 1: intention, selfregulatory capacity and interactions between intention and self-regulatory capacity variables as predictors of sunscreen use, $\mathrm{N}=209$.

\begin{tabular}{|c|c|c|c|c|c|c|}
\hline Model & Variable & $\beta$ & $t$ & $R^{2}$ & $\Delta R^{2}$ & $p$ \\
\hline \multirow[t]{8}{*}{ Step 6} & INT & .26 & 3.82 & & & $<.01 * *$ \\
\hline & TOL & -.01 & -.13 & & & .90 \\
\hline & WCST & -.05 & -.79 & & & .43 \\
\hline & IGT & .01 & .21 & & & .84 \\
\hline & INTxTOI & -.04 & -.55 & & & .58 \\
\hline & INTxWC & .02 & .28 & & & .78 \\
\hline & INTxIGT & .03 & .38 & & & .71 \\
\hline & & & & .075 & .001 & .70 \\
\hline
\end{tabular}

Note: INT = intention, TOL = planning ability measured by average time spent preplanning (seconds) on Tower of London Task, WCST = cognitive flexibility measured by number of perseverance errors on the Wisconsin Card Sort Task, IGT = impulsivity measured by remaining play money (\$) on Iowa Gambling Task, INTxTOL = interaction between intention and planning ability, INTxWCST = interaction between intention and cognitive flexibility, INTxIGT = interaction between intention and impulsivity, ${ }^{* *} p<.01$ 
Table 3. Descriptive analysis of sun protection behaviour for Study 2: means, standard deviations and bivariate correlations, $\mathrm{N}=178$.

\begin{tabular}{cccccccccc}
\hline & $M$ & SD & INT & PB & TOL & WCST & IGT & HAB & SPB \\
\hline INT & 3.75 & 1.71 & - & $.37^{* *}$ & -.06 & $-.16^{*}$ & .04 & $.49^{* *}$ & $.49^{* *}$ \\
PB & 3.38 & 1.69 & - & - & -.06 & $-.17^{*}$ & .03 & $.55^{* *}$ & $.48^{* *}$ \\
TOL & 2.69 & 1.04 & - & - & - & .02 & -.09 & -.06 & -.01 \\
WCST & 49.85 & 23.00 & - & - & - & - & -.05 & $-.27^{* *}$ & $-.43^{* *}$ \\
IGT & 1719.66 & 673.57 & - & - & - & - & - & -.01 & -.01 \\
HAB & 3.12 & 1.59 & - & - & - & - & - & - & $.64^{* *}$ \\
SPB & .48 & .32 & - & - & - & - & - & - & - \\
\hline
\end{tabular}

Note: $\mathrm{INT}=$ intention, $\mathrm{PB}$ = past behaviour; $\mathrm{TOL}$ = planning ability measured by average time spent preplanning (seconds) on Tower of London Task, WCST = cognitive flexibility measured by number of perseverance errors on the Wisconsin Card Sort Task, IGT = impulsivity measured by remaining play money (\$) on Iowa Gambling Task, HAB = habit as measured by the Self-Report Habit Index; SPB = proportion of times used sun protection behaviour when in sun; * $p<.05$; level, $* * p<.01$. 
Table 4. Final step of hierarchical regression analysis for Study 2: intention, past behaviour, cognitive flexibility, habit and interactions between intention and cognitive flexibility and intention and habit as predictors of sun protection behaviours, $\mathrm{N}=178$.

\begin{tabular}{|c|c|c|c|c|c|c|}
\hline Model & Variable & $\beta$ & $t$ & $R^{2}$ & $\Delta R^{2}$ & $p$ \\
\hline \multirow[t]{7}{*}{ Step 6} & INT & .23 & 3.85 & & & $<.01 * *$ \\
\hline & PB & .19 & 3.06 & & & $<.01 * *$ \\
\hline & WCST & -.28 & -5.20 & & & $<.01 * *$ \\
\hline & HAB & .36 & 5.28 & & & $<.01 * *$ \\
\hline & INTxWCST & .08 & 1.52 & & & .131 \\
\hline & INTxHAB & -.15 & -2.84 & & & $<.01 * *$ \\
\hline & & & & .561 & .021 & .005 \\
\hline
\end{tabular}

Note: INT = intention, $\mathrm{PB}$ = past behaviour; WCST = cognitive flexibility measured by number of perseverance errors on the Wisconsin Card Sort Task, HAB = habit as measured by the Self-Report Habit Index; INTxWCST = interaction between intention and cognitive flexibility, INTxHAB = interaction between intention and habit, overall $R^{2}=.561 ; * * p<.01$. 


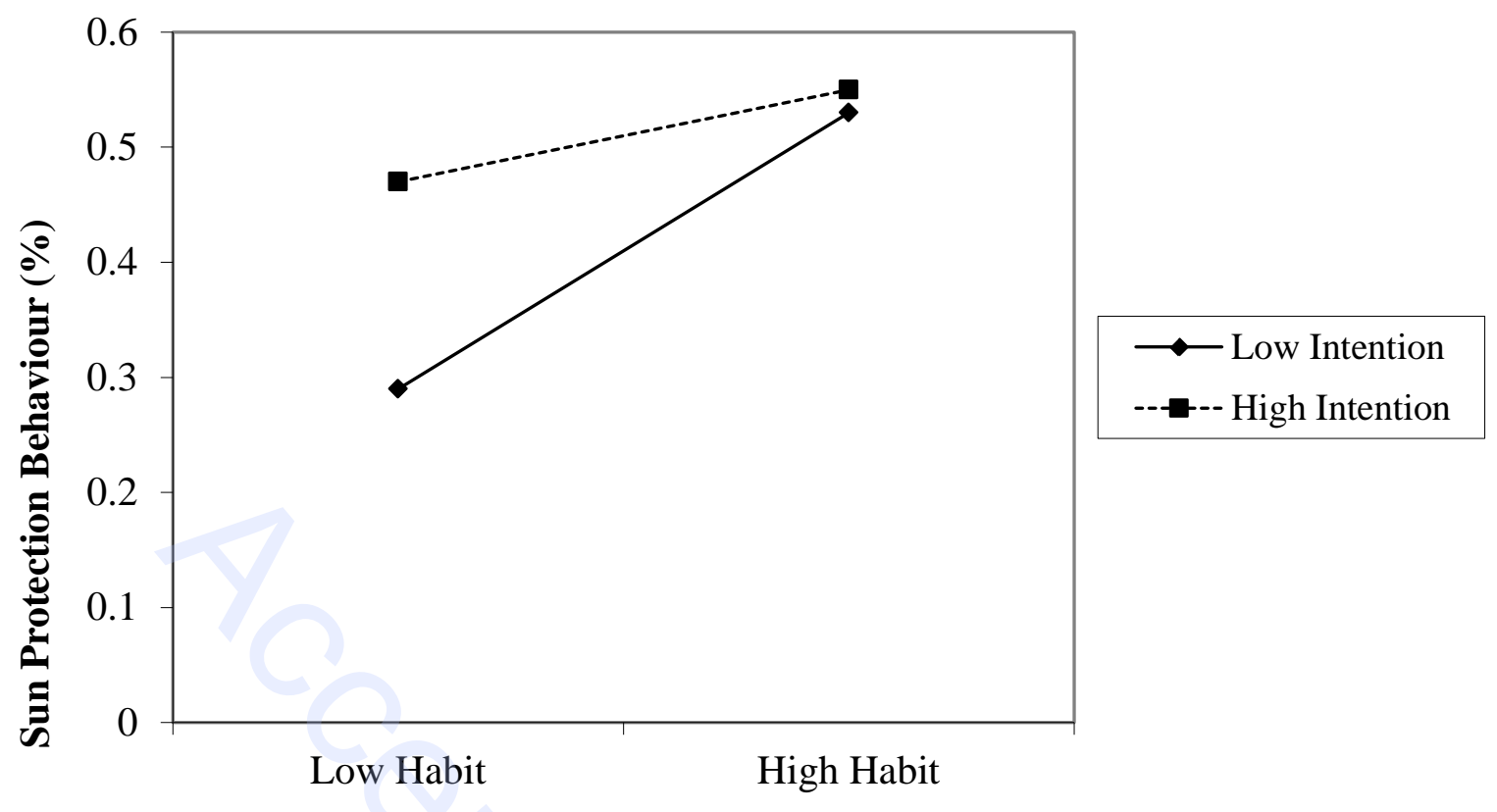




\section{Figure Caption}

Figure 1. Moderating effect of habit on intention and sun protection behaviour (\% of times used when in sun). 